
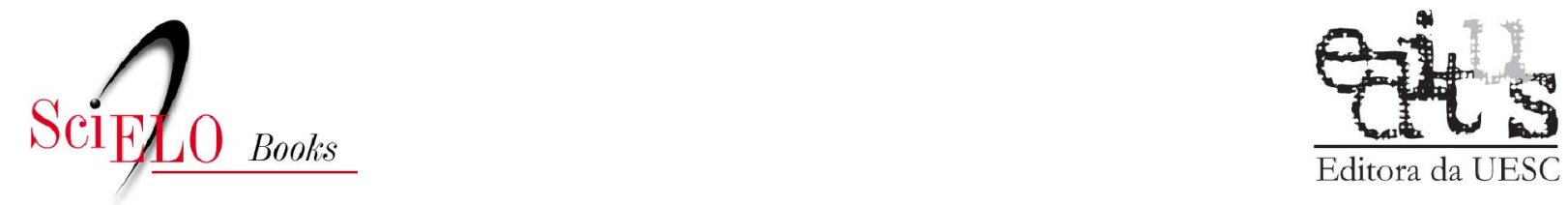

Editora da UESC

\title{
9 - Lista das Formigas Poneromorfas do Brasil
}

\author{
Rodrigo M. Feitosa
}

SciELO Books / SciELO Livros / SciELO Libros

FEITOSA, RM. Lista das Formigas Poneromorfas do Brasil. In: DELABIE, JHC., et al., orgs. As formigas poneromorfas do Brasil [online]. Ilhéus, BA: Editus, 2015, pp. 95-101. ISBN 978-85-7455441-9. Available from SciELO Books $<\underline{\text { http://books.scielo.org }>\text {. }}$

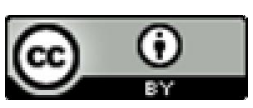

All the contents of this work, except where otherwise noted, is licensed under a Creative Commons Attribution $\underline{4.0 \text { International license. }}$

Todo o conteúdo deste trabalho, exceto quando houver ressalva, é publicado sob a licença Creative Commons Atribição 4.0.

Todo el contenido de esta obra, excepto donde se indique lo contrario, está bajo licencia de la licencia Creative Commons Reconocimento 4.0. 


\title{
Lista das Formigas Poneromorfas do Brasil
}

\author{
Rodrigo M. Feitosa
}

\section{Resumo}

Com cerca de 13.000 espécies descritas em todo mundo, formigas representam o grupo mais especioso e ecologicamente diverso de insetos sociais. O Brasil, com seus seis biomas distribuídos em continentais $8.500 .000 \mathrm{~km}^{2}$ de território, abriga a maior diversidade de gêneros de formigas e a segunda maior diversidade de espécies do mundo. Embora estes dados sejam representativos, o conhecimento sobre a diversidade de formigas no Brasil ainda está longe de estar completo. Quando comparamos o nível de conhecimento taxonômico entre os táxons de Formicidae que ocorrem no Brasil, as subfamílias poneromorfas encontram-se em um estágio mais avançado que as demais, especialmente quando comparadas aos gêneros mais diversos de Formicinae e Myrmicinae. Neste capítulo é apresentada uma lista sinóptica das espécies de formigas poneroides e ectatomminoides que ocorrem no Brasil.

FEITOSA, Rodrigo M. Lista das Formigas Poneromorfas do Brasil. In: DELABIE, Jacques H. C. et al. As formigas poneromorfas do Brasil. Ilhéus: Editus, 2015. p. 95-101. 


\section{Abstract}

List of Brazilian poneromorph ants - With about 13,000 species described worldwide, ants are the most speciose and ecologically diverse group of social insects. Brazil, with its six biomes distributed across an impressive $8,500,000 \mathrm{~km}^{2}$ of territory, is home to the greatest diversity of ant genera and the second highest ant species diversity in the world. Although these data are impressive, knowledge about ant diversity in Brazil is still far from complete. When comparing the level of taxonomic knowledge among the Formicidae taxa that occur in Brazil, poneromorph subfamilies are at a more advanced stage than the others, especially when compared to the most diverse genera in Formicinae and Myrmicinae. In this chapter, a synoptic list of species of poneromorph ants occurring in Brazil is presented.

\section{Introdução}

De acordo com o último catálogo taxonômico para formigas (BOLTON, 2015), Formicidae soma 16 subfamílias atuais e quatro extintas, englobando aproximadamente 13.000 espécies descritas em cerca de 330 gêneros viventes até o ano de 2014. Contudo, esses números são constantemente alterados. Por um lado, enquanto o conhecimento taxonômico acerca das formigas aumenta, muito dos nomes específicos e subespecíficos propostos mostram-se redundantes (sinônimos). Por outro lado, é evidente que há muitas espécies a serem descobertas e/ou descritas. Em trabalhos taxonômicos recentes, o número de espécies sinonimizadas tem sido sempre menor que o número de espécies descritas, em alguns casos por uma ampla margem (BOLTON, 2000; WILSON, 2003; BOLTON; FISHER, 2011). Estimativas sugerem que o número total de espécies de formigas atuais supere 25.000, sendo que a maior parte do contingente ainda não descrito concentra-se nas florestas tropicais (FERNÁNDEZ; OSPINA, 2003; LACH; et al., 2010).

Com uma extensão territorial de cerca de $8.500 .000 \mathrm{~km}^{2}$, o Brasil ocupa sozinho quase $50 \%$ da área total da América do Sul, com a maior parte de seu território inserida na região tropical do planeta. Esta superfície de proporções continentais é em grande parte responsável pela grande heterogeneidade de ambientes naturais encontrada no país. Variações em fatores como latitude, topografia, clima e conformação de bacias hidrográficas proporcionaram a formação dos seis biomas terrestres brasileiros (Amazônia, Caatinga, Cerrado, Mata Atlântica, Pampas e Pantanal), que por sua vez são divididos em distintas fisionomias. É justamente esta heterogeneidade que faz com que o Brasil seja o país que concentra a maior biodiversidade do planeta.

As formigas não são uma exceção e, atualmente, o Brasil concentra a maior diversidade de gêneros e a segunda maior diversidade de espécies de formigas do mundo (BOLTON, 2015). No Brasil ocorrem 108 dos 324 gêneros reconhecidos para formigas em todo o planeta até a presente data, sendo que nove destes gêneros não são encontrados em nenhum outro país (Tabela 9.I). Ainda, o Brasil abriga cerca de 1.460 espécies e subespécies de formigas, das quais $37 \%$ (aprox. 540) são endêmicas. Este número de espécies só é menor do que aquele encontrado na Austrália (cerca de 1.520 espécies). Ainda assim, com o incremento recente do número de estudos sobre as formigas brasileiras, é muito provável que em breve o Brasil assuma a posição de país com a maior diversidade de formigas do planeta.

Todas as seis subfamílias conhecidas de formigas poneroides estão presentes no Brasil, representadas por 26 gêneros e 214 espécies. A lista apresentada neste capítulo (Tabela 9.II) baseia-se primariamente no levantamento feito por Fernández; Arias-Penna (2007) para as espécies de formigas caçadoras da Região Neotropical. Os dados foram atualizados e complementados de acordo com estudos taxonômicos publicados recentemente. A classificação adotada segue Bolton (2015).

A recente publicação de Schmidt; Shattuck (2014) oferece uma classificação da subfamília Ponerinae com base em uma abrangente filogenia molecular. Para a fauna do Brasil e dos neotrópicos 
o gênero Pachycondyla é dividido nos seguintes gêneros: Cryptopone Emery, Mayaponera Schmidt; Shattuck, Neoponera Emery, Pachycondyla Smith, Pseudoponera Emery e Rasopone Schmidt; Shattuck. Na presente lista apresentamos a classificação de Ponerinae já no conceito de Schmidt;
Shattuck (2014). No entanto, ao buscar informações sobre taxonomia e distribuição das espécies dos gêneros mencionados acima, o leitor deve considerar consultar o trabalho de Mackay; Mackay (2010) com a revisão taxonômica do gênero Pachycondyla s. l. para o Novo Mundo.

Tabela 9.II - Lista comparativa da diversidade de espécies e gêneros de formigas poneromorfas no Brasil, Região Neotropical e no mundo

\begin{tabular}{|c|c|c|c|c|c|c|}
\hline \multirow[t]{2}{*}{ TÁXON } & \multicolumn{3}{|c|}{ ESPÉCIES } & \multicolumn{3}{|c|}{ GÊNEROS } \\
\hline & Brasil & Neotrópicos & Mundo & Brasil & Neotrópicos & Mundo \\
\hline Amblyoponinae & 11 & 17 & 125 & 2 & 3 & 13 \\
\hline Prionopelta & 3 & 4 & 15 & & & \\
\hline Stigmatomma & 8 & 12 & 68 & & & \\
\hline Ectatomminae & 50 & 113 & 267 & 3 & 3 & 4 \\
\hline Ectatomma & 11 & 15 & 15 & & & \\
\hline Gnamptogenys & 33 & 90 & 140 & & & \\
\hline Typhlomyrmex & 6 & 7 & 7 & & & \\
\hline Heteroponerinae & 11 & 16 & 24 & 2 & 2 & 3 \\
\hline Acanthoponera & 3 & 4 & 4 & & & \\
\hline Heteroponera & 8 & 12 & 19 & & & \\
\hline Paraponerinae & 1 & 1 & 1 & 1 & 1 & 1 \\
\hline Paraponera & 1 & 1 & 1 & & & \\
\hline Ponerinae & 135 & 341 & 1061 & 15 & 17 & 47 \\
\hline Anochetus & 10 & 27 & 114 & & & \\
\hline Centromyrmex & 3 & 3 & 15 & & & \\
\hline Cryptopone & 2 & 4 & 25 & & & \\
\hline Dinoponera & 8 & 8 & 8 & & & \\
\hline Hypoponera & 17 & 35 & 147 & & & \\
\hline Leptogenys & 19 & 75 & 265 & & & \\
\hline Mayaponera & 1 & 1 & 1 & & & \\
\hline Neoponera & 35 & 54 & 54 & & & \\
\hline Odontomachus & 15 & 24 & 66 & & & \\
\hline Pachycondyla & 8 & 11 & 11 & & & \\
\hline Pseudoponera & 3 & 5 & 6 & & & \\
\hline Rasopone & 3 & 11 & 11 & & & \\
\hline Simopelta & 4 & 12 & 21 & & & \\
\hline Platythyrea & 5 & 8 & 39 & & & \\
\hline Thaumatomyrmex & 2 & 12 & 12 & & & \\
\hline Proceratiinae & 6 & 25 & 133 & 3 & 3 & 3 \\
\hline Probolomyrmex & 3 & 4 & 24 & & & \\
\hline Discothyrea & 2 & 7 & 32 & & & \\
\hline Proceratium & 1 & 15 & 77 & & & \\
\hline Poneromorfas & 214 & 513 & 1611 & 26 & 29 & 71 \\
\hline
\end{tabular}

TABELA 9.II - Lista taxonômica das espécies poneromorfas de formigas do Brasil

\begin{tabular}{l|l}
\hline TÁXON & REFERÊNCIA \\
\hline Subfamília Amblyoponinae & \\
\hline Tribo Amblyoponini & \\
\hline Prionopelta antillana Forel, 1909 & Brown, 1960 \\
\hline Prionopelta modesta Forel, 1909 & Brown, 1960 \\
\hline Prionopelta punctulata Mayr, 1866 & Brown, 1960 \\
\hline Stigmatomma agostii (Lacau; Delabie, 2002) & Lacau; Delabie, 2002 \\
\hline Stigmatomma armigerum (Mayr, 1887) & Lattke, 1991 \\
\hline Stigmatomma cleae (Lacau; Delabie, 2002) & Lacau; Delabie, 2002 \\
\hline Stigmatomma degeneratum (Borgmeier, 1957) & Lattke, 1991 \\
\hline Stigmatomma elongatum Santschi, 1912 & Lattke, 1991 \\
\hline Stigmatomma heraldoi (Lacau; Delabie, 2002) & Lacau; Delabie, 2002 \\
\hline Stigmatomma lurilabes (Lattke, 1991) & Lattke, 1991 \\
\hline Stigmatomma mystriops (Brown, 1960) & Lattke, 1991 \\
\hline
\end{tabular}

\begin{tabular}{l|l}
\hline TÁXON & REFERÊNCIA \\
\hline Subfamília Ectatomminae & \\
\hline Tribo Ectatommini & \\
\hline Ectatomma brunneum Smith, F., 1858 & Kugler; Brown, 1982 \\
\hline Ectatomma edentatum Roger, 1863 & Kugler; Brown, 1982 \\
\hline Ectatomma lugens Emery, 1894 & Kugler; Brown, 1982 \\
\hline Ectatomma muticum Mayr, 1870 & Kugler; Brown, 1982 \\
\hline Ectatomma opaciventre (Roger, 1861) & Kugler; Brown, 1982 \\
\hline Ectatomma permagnum Forel, 1908 & Kugler; Brown, 1982 \\
\hline Ectatomma planidens Borgmeier, 1939 & Kugler; Brown, 1982 \\
\hline Ectatomma ruidum (Roger, 1860) & Kugler; Brown, 1982 \\
\hline Ectatomma suzanae Almeida, 1986 & Almeida, 1986 \\
\hline Ectatomma tuberculatum (Olivier, 1792) & Kugler; Brown, 1982 \\
\hline Ectatomma vizottoi Almeida, 1987 & Almeida, 1987 \\
\hline
\end{tabular}




\begin{tabular}{|c|c|}
\hline TÁXON & REFERÊNCIA \\
\hline Gnamptogenys acuminata (Emery, 1896) & Lattke, 1995 \\
\hline Gnamptogenys annulata (Mayr, 1887) & Lattke, 1995 \\
\hline Gnamptogenys bruchi (Santschi, 1922) & Lattke, 1995 \\
\hline Gnamptogenys caelata Kempf, 1967 & Lattke, 1995 \\
\hline Gnamptogenys concinna (Smith, F., 1858) & Lattke, 1995 \\
\hline Gnamptogenys continua (Mayr, 1887) & Lattke, 1995 \\
\hline Gnamptogenys haenschi (Emery, 1902) & Lattke, 1995 \\
\hline Gnamptogenys horni (Santschi, 1929) & Lattke, 1995 \\
\hline Gnamptogenys interrupta (Mayr, 1887) & Lattke, 1995 \\
\hline Gnamptogenys kempfi Lenko, 1964 & Lattke, 1995 \\
\hline Gnamptogenys lanei Kempf, 1960 & Lattke, 1995 \\
\hline Gnamptogenys lavra Lattke, 2002 & Lattke, 2002 \\
\hline Gnamptogenys lucaris Kempf, 1968 & Lattke, 1995 \\
\hline Gnamptogenys mecotyle Brown, 1958 & Lattke, 1995 \\
\hline Gnamptogenys mediatrix Brown, 1958 & Lattke, 1995 \\
\hline Gnamptogenys menozzii Borgmeier, 1928 & Lattke, 1995 \\
\hline Gnamptogenys minuta (Emery, 1896) & Lattke, 1995 \\
\hline Gnamptogenys moelleri (Forel, 1912) & Lattke, 1995 \\
\hline Gnamptogenys mordax (Smith, F., 1858) & Lattke, 1995 \\
\hline Gnamptogenys nana Kempf, 1960 & Lattke, 1995 \\
\hline Gnamptogenys pleurodon (Emery, 1896) & Lattke, 1995 \\
\hline Gnamptogenys porcata (Emery, 1896) & Lattke, 1995 \\
\hline Gnamptogenys rastrata (Mayr, 1866) & Lattke, 1995 \\
\hline Gnamptogenys regularis Mayr, 1870 & Lattke, 1995 \\
\hline Gnamptogenys reichenspergeri (Santschi, 1929) & Lattke, 1995 \\
\hline Gnamptogenys relicta (Mann, 1916) & Lattke, 1995 \\
\hline Gnamptogenys rimulosa (Roger, 1861) & Lattke, 1995 \\
\hline Gnamptogenys striatula (Mayr, 1884) & Lattke, 1995 \\
\hline Gnamptogenys striolata Borgmeier, 1957 & Lattke, 1995 \\
\hline Gnamptogenys sulcata (Smith, F., 1858) & Lattke, 1995 \\
\hline Gnamptogenys tortuolosa (Smith, F., 1858) & Lattke, 1995 \\
\hline Gnamptogenys triangularis (Mayr, 1887) & Lattke, 1995 \\
\hline Gnamptogenys wilsoni Lattke, 2007 & Lattke et al., 2007 \\
\hline \multicolumn{2}{|l|}{ Tribo Typhlomyrmecini } \\
\hline Typhlomyrmex clavicornis Emery, 1906 & Brown, 1965 \\
\hline Typhlomyrmex foreli Santschi, 1925 & Brown, 1965 \\
\hline Typhlomyrmex major Santschi, 1923 & Brown, 1965 \\
\hline $\begin{array}{l}\text { Typhlomyrmex meire Lacau, Villemant; Delabie, } \\
2004\end{array}$ & Lacau et al., 2008 \\
\hline Typhlomyrmex pusillus Emery, 1894 & Brown, 1965 \\
\hline Typhlomyrmex rogenhoferi Mayr, 1862 & Brown, 1965 \\
\hline \multicolumn{2}{|l|}{ Subfamília Heteroponerinae } \\
\hline \multicolumn{2}{|l|}{ Tribo Heteroponerini } \\
\hline Acanthoponera goeldii Forel, 1912 & Brown, 1958 \\
\hline Acanthoponera mucronata (Roger, 1860) & Brown, 1958 \\
\hline Acanthoponera peruviana Brown, 1958 & Brown, 1958 \\
\hline Heteroponera angulata Borgmeier, 1959 & Kempf, 1962 \\
\hline Heteroponera dentinodis (Mayr, 1887) & Kempf, 1962 \\
\hline Heteroponera dolo (Roger, 1860) & Kempf, 1962 \\
\hline Heteroponera flava Kempf, 1962 & Kempf, 1962 \\
\hline Heteroponera inermis (Emery, 1894) & Kempf, 1962 \\
\hline Heteroponera mayri Kempf, 1962 & Kempf, 1962 \\
\hline Heteroponera microps Borgmeier, 1957 & Kempf, 1962 \\
\hline Heteroponera robusta Kempf, 1962 & Kempf, 1962 \\
\hline \multicolumn{2}{|l|}{ Subfamília Paraponerinae } \\
\hline \multicolumn{2}{|l|}{ Tribo Paraponerini } \\
\hline Paraponera clavata (Fabricius, 1775) & Brown, 1958 \\
\hline
\end{tabular}

\begin{tabular}{|c|c|}
\hline TÁXON & REFERÊNCIA \\
\hline \multicolumn{2}{|l|}{ Subfamília Ponerinae } \\
\hline \multicolumn{2}{|l|}{ Tribo Ponerini } \\
\hline Anochetus altisquamis Mayr, 1887 & Brown, 1978 \\
\hline Anochetus bispinosus (Smith, F., 1858) & Brown, 1978 \\
\hline Anochetus diegensis Forel, 1912 & Brown, 1978 \\
\hline Anochetus hohenbergiae Feitosa; Delabie, 2012 & Feitosa et al., 2012 \\
\hline Anochetus horridus Kempf, 1964 & Brown, 1978 \\
\hline Anochetus mayri Emery, 1884 & Brown, 1978 \\
\hline Anochetus neglectus Emery, 1894 & Brown, 1978 \\
\hline Anochetus oriens Kempf, 1964 & Brown, 1978 \\
\hline Anochetus targionii Emery, 1894 & Brown, 1978 \\
\hline Anochetus vexator Kempf, 1964 & Brown, 1978 \\
\hline Centromyrmex alfaroi Emery, 1890 & Kempf, 1967 \\
\hline Centromyrmex brachycola (Roger, 1861) & Kempf, 1967 \\
\hline Centromyrmex gigas Forel, 1911 & Kempf, 1967 \\
\hline Cryptopone guianensis (Weber, 1939) & Mackay; Mackay, 2010 \\
\hline Cryptopone holmgreni (Wheeler, 1925) & Mackay; Mackay, 2010 \\
\hline Dinoponera australis Emery, 1901 & Lenhart et al., 2013 \\
\hline Dinoponera gigantea (Perty, 1833) & Lenhart et al., 2013 \\
\hline Dinoponera hispida Lenhart, Dash; Mackay, 2013 & Lenhart et al., 2013 \\
\hline Dinoponera longipes Emery, 1901 & Lenhart et al., 2013 \\
\hline Dinoponera lucida Emery, 1901 & Lenhart et al., 2013 \\
\hline Dinoponera mutica Emery, 1901 & Lenhart et al., 2013 \\
\hline Dinoponera quadriceps Kempf, 1971 & Lenhart et al., 2013 \\
\hline Dinoponera snellingi Lenhart, Dash; Mackay, 2013 & Lenhart et al., 2013 \\
\hline Hypoponera aliena (Smith, F., 1858) & Kempf, 1972 \\
\hline Hypoponera collegiana (Santschi, 1925) & Kempf, 1972 \\
\hline Hypoponera collegiana paranensis (Santschi, 1925) & Kempf, 1972 \\
\hline Hypoponera distinguenda (Emery, 1980) & Kempf, 1972 \\
\hline Hypoponera distinguenda dispar (Santschi, 1925) & Kempf, 1972 \\
\hline Hypoponera distinguenda histrio (Forel, 1912) & Kempf, 1972 \\
\hline Hypoponera distinguenda inexpedita (Forel, 1911) & Kempf, 1972 \\
\hline Hypoponera foeda saroltae (Forel, 1912) & Kempf, 1972 \\
\hline Hypoponera foreli (Mayr, 1887) & Kempf, 1972 \\
\hline Hypoponera idelettae (Santschi, 1923) & Kempf, 1972 \\
\hline Hypoponera iheringi (Forel, 1908) & Kempf, 1972 \\
\hline Hypoponera leninei (Santschi, 1925) & Kempf, 1972 \\
\hline Hypoponera neglecta (Santschi, 1923) & Kempf, 1972 \\
\hline Hypoponera opaciceps (Mayr, 1887) & Kempf, 1972 \\
\hline Hypoponera reichenspergeri (Santschi, 1923) & Kempf, 1972 \\
\hline Hypoponera schmalzi (Forel, 1912) & Kempf, 1972 \\
\hline Hypoponera schmalzi paulina (Forel, 1913) & Kempf, 1972 \\
\hline Hypoponera schmalzi fugitans (Forel, 1912) & Kempf, 1972 \\
\hline Hypoponera schwebeli (Forel, 1913) & Kempf, 1972 \\
\hline Hypoponera trigona (Mayr, 1887) & Kempf, 1972 \\
\hline Hypoponera trigona cauta (Forel, 1912) & Kempf, 1972 \\
\hline Hypoponera vernacula (Kempf, 1962) & Kempf, 1972 \\
\hline Hypoponera viri (Santschi, 1923) & Kempf, 1972 \\
\hline Hypoponera wilsoni (Santschi, 1925) & Kempf, 1972 \\
\hline Leptogenys amazonica Borgmeier, 1930 & Lattke, 2011 \\
\hline Leptogenys arcuata Roger, 1861 & Lattke, 2011 \\
\hline Leptogenys australis (Emery, 1888) & Lattke, 2011 \\
\hline Leptogenys bohlsi Emery, 1896 & Lattke, 2011 \\
\hline Leptogenys corniculans Lattke, 2011 & Lattke, 2011 \\
\hline Leptogenys crudelis (Smith, F., 1858) & Lattke, 2011 \\
\hline Leptogenys gaigei Wheeler, 1923 & Lattke, 2011 \\
\hline Leptogenys guianensis Wheeler, 1923 & Lattke, 2011 \\
\hline
\end{tabular}




\begin{tabular}{|c|c|}
\hline TÁXON & REFERÊNCIA \\
\hline Leptogenys iheringi Forel, 1911 & Lattke, 2011 \\
\hline Leptogenys linearis (Smith, F., 1858) & Lattke, 2011 \\
\hline Leptogenys langi Wheeler, 1923 & Lattke, 2011 \\
\hline Leptogenys luederwaldti Forel, 1913 & Lattke, 2011 \\
\hline Leptogenys mavaca Lattke, 2011 & Lattke, 2011 \\
\hline Leptogenys maxillosa (Smith, F. 1858) & Lattke, 2011 \\
\hline Leptogenys minima Lattke, 2011 & Lattke, 2011 \\
\hline Leptogenys nigricans Lattke, 2011 & Lattke, 2011 \\
\hline Leptogenys paraense Lattke, 2011 & Lattke, 2011 \\
\hline Leptogenys unistimulosa Roger, 1863 & Lattke, 2011 \\
\hline Leptogenys vogeli Borgmeier, 1933 & Lattke, 2011 \\
\hline Mayaponera constricta (Mayr, 1884) & Mackay; Mackay, 2010 \\
\hline Neoponera agilis Forel, 1901 & Mackay; Mackay, 2010 \\
\hline Neoponera antecurvata (Mackay; Mackay, 2010) & Mackay; Mackay, 2010 \\
\hline Neoponera apicalis (Latreille, 1802) & Mackay; Mackay, 2010 \\
\hline Neoponera bactronica (Fernandes; Delabie, 2014) & Fernandes et al., 2014 \\
\hline Neoponera billemma (Fernandes; Delabie, 2014) & Fernandes et al., 2014 \\
\hline Neoponera bucki (Borgmeier, 1927) & Mackay; Mackay, 2010 \\
\hline Neoponera carinulata (Roger, 1861) & Mackay; Mackay, 2010 \\
\hline Neoponera cavinodis (Mann, 1916) & Mackay; Mackay, 2010 \\
\hline Neoponera commutata (Roger, 1860) & Mackay; Mackay, 2010 \\
\hline Neoponera concava (Mackay; Mackay, 2010) & Mackay; Mackay, 2010 \\
\hline Neoponera cooki (Mackay; Mackay, 2010) & Mackay; Mackay, 2010 \\
\hline Neoponera crenata (Roger, 1861) & Mackay; Mackay, 2010 \\
\hline Neoponera curvinodis (Forel, 1899) & Mackay; Mackay, 2010 \\
\hline Neoponera foetida (Linnaeus, 1758) & Mackay; Mackay, 2010 \\
\hline Neoponera goeldii Forel, 1912 & Mackay; Mackay, 2010 \\
\hline Neoponera inversa (Smith, F., 1858) & Mackay; Mackay, 2010 \\
\hline Neoponera laevigata (Smith, F., 1858) & Mackay; Mackay, 2010 \\
\hline Neoponera latinoda (Mackay; Mackay, 2010) & Mackay; Mackay, 2010 \\
\hline Neoponera luteola (Roger, 1861) & Mackay; Mackay, 2010 \\
\hline Neoponera magnifica (Borgmeier, 1929) & Mackay; Mackay, 2010 \\
\hline Neoponera marginata (Roger, 1861) & Mackay; Mackay, 2010 \\
\hline Neoponera metanotalis (Luederwaldt, 1918) & Mackay; Mackay, 2010 \\
\hline Neoponera moesta (Mayr, 1870) & Mackay; Mackay, 2010 \\
\hline Neoponera oberthueri (Emery, 1890) & Mackay; Mackay, 2010 \\
\hline Neoponera obscuricornis (Emery, 1890) & Mackay; Mackay, 2010 \\
\hline Neoponera procidua Emery, 1890 & Mackay; Mackay, 2010 \\
\hline Neoponera rostrata (Emery, 1890) & Mackay; Mackay, 2010 \\
\hline Neoponera rugosula (Emery, 1902) & Mackay; Mackay, 2010 \\
\hline Neoponera schultzi (Mackay; Mackay, 2010) & Mackay; Mackay, 2010 \\
\hline Neoponera striatinodis (Emery, 1890) & Mackay; Mackay, 2010 \\
\hline Neoponera theresiae (Forel, 1899) & Mackay; Mackay, 2010 \\
\hline Neoponera unidentata (Mayr, 1862) & Mackay; Mackay, 2010 \\
\hline Neoponera venusta (Forel, 1912) & Mackay; Mackay, 2010 \\
\hline Neoponera verenae Forel, 1922 & Mackay; Mackay, 2010 \\
\hline Neoponera villosa (Fabricius, 1804) & Mackay; Mackay, 2010 \\
\hline Odontomachus affinis Guerin-Meneville, 1844 & Brown, 1976 \\
\hline Odontomachus allolabis Kempf, 1974 & Brown, 1976 \\
\hline Odontomachus bauri Emery, 1892 & Brown, 1976 \\
\hline Odontomachus biumbonatus Brown, 1976 & Brown, 1976 \\
\hline Odontomachus brunneus (Patton, 1894) & Brown, 1976 \\
\hline Odontomachus caelatus Brown, 1976 & Brown, 1976 \\
\hline Odontomachus chelifer (Latreille, 1802) & Brown, 1976 \\
\hline Odontomachus haematodus (Linnaeus, 1758) & Brown, 1976 \\
\hline Odontomachus hastatus (Fabricius, 1804) & Brown, 1976 \\
\hline Odontomachus laticeps Roger, 1861 & Brown, 1976 \\
\hline Odontomachus mayi Mann, 1912 & Brown, 1976 \\
\hline Odontomachus meinerti Forel, 1905 & Brown, 1976 \\
\hline
\end{tabular}

\begin{tabular}{|c|c|}
\hline TÁXON & REFERÊNCIA \\
\hline Odontomachus opaciventris Forel, 1899 & Brown, 1976 \\
\hline Odontomachus panamensis Forel, 1899 & Brown, 1976 \\
\hline Odontomachus spissus Kempf, 1962 & Brown, 1976 \\
\hline Pachycondyla crassinoda (Latreille, 1802) & Mackay; Mackay, 2010 \\
\hline Pachycondyla curiosa Mackay; Mackay, 2010 & Mackay; Mackay, 2010 \\
\hline Pachycondyla fuscoatra (Roger, 1861) & Mackay; Mackay, 2010 \\
\hline Pachycondyla harpax (Fabricius, 1804) & Mackay; Mackay, 2010 \\
\hline Pachycondyla impressa (Roger, 1861) & Mackay; Mackay, 2010 \\
\hline Pachycondyla lenis Kempf, 1961 & Mackay; Mackay, 2010 \\
\hline Pachycondyla lenkoi Kempf, 1962 & Mackay; Mackay, 2010 \\
\hline Pachycondyla striata Smith, F., 1858 & Mackay; Mackay, 2010 \\
\hline Pseudoponera gilberti (Kempf, 1960) & Mackay; Mackay, 2010 \\
\hline Pseudoponera stigma (Fabricius, 1804) & Mackay; Mackay, 2010 \\
\hline Pseudoponera succedanea (Roger, 1863) & Mackay; Mackay, 2010 \\
\hline Rasopone arhuaca (Forel, 1901) & Mackay; Mackay, 2010 \\
\hline Rasopone ferruginea (Smith, F., 1858) & Mackay; Mackay, 2010 \\
\hline Rasopone lunaris (Emery, 1896) & Mackay; Mackay, 2010 \\
\hline Simopelta bicolor Borgmeier, 1950 & Mackay; Mackay, 2008 \\
\hline Simopelta curvata (Mayr, 1887) & Mackay; Mackay, 2008 \\
\hline Simopelta jeckylli (Mann, 1916) & Mackay; Mackay, 2008 \\
\hline Simopelta minima (Brandão, 1989) & Mackay; Mackay, 2008 \\
\hline Thaumatomyrmex contumax Kempf, 1975 & Jahyny et al., 2007 \\
\hline Thaumatomyrmex mutilatus Mayr, 1887 & Jahyny et al., 2007 \\
\hline \multicolumn{2}{|l|}{ Tribo Platythyreini } \\
\hline Platythyrea angusta Forel, 1901 & Brown, 1975 \\
\hline Platythyrea exigua Kempf, 1964 & Brown, 1975 \\
\hline Platythyrea pilosula (Smith, F., 1858) & Brown, 1975 \\
\hline Platythyrea punctata (Smith, F., 1858) & Brown, 1975 \\
\hline Platythyrea sinuata (Roger, 1860) & Brown, 1975 \\
\hline \multicolumn{2}{|l|}{ Subfamília Proceratiinae } \\
\hline \multicolumn{2}{|l|}{ Tribo Probolomyrmecini } \\
\hline Probolomyrmex boliviensis Mann, 1923 & Agosti, 1995 \\
\hline Probolomyrmex brujitae Agosti, 1995 & Agosti, 1995 \\
\hline Probolomyrmex petiolatus Weber, 1940 & Agosti, 1995 \\
\hline \multicolumn{2}{|l|}{ Tribo Proceratiini } \\
\hline Discothyrea neotropica Borgmeier, 1953 & $\begin{array}{l}\text { Sosa-Calvo; Longino, } \\
2007\end{array}$ \\
\hline Discothyrea sexarticulata Bruch, 1919 & $\begin{array}{l}\text { Sosa-Calvo; Longino, } \\
2007\end{array}$ \\
\hline Proceratium brasiliense Borgmeier, 1959 & $\begin{array}{l}\text { Baroni-Urbani; de } \\
\text { Andrade, } 2003\end{array}$ \\
\hline
\end{tabular}

\section{Referências}

AGOSTI, D. A revision of the South American species of the ant genus Probolomyrmex (Hymenoptera: Formicidae). Journal of the New York Entomological Society, v.102, Published: 19 Jul 1995, p.429-434, 1995 (1994).

ALMEIDA FILHO, A. J. D. Descrição de quatro machos do gênero Ectatomma Smith, 1858 (Hymenoptera, Formicidae, Ponerinae). Quid (Teresina), v.6, Published: Dec 1986, p.24-38, 1986.

ALMEIDA FILHO, A. J. D. Descrição de seis fêmeas do gênero Ectatomma Smith, 1858 (Hymenoptera, Formicidae, Ponerinae). Museo Regionale di Scienze Naturali Monografie (Turin), v.1, p.175183, 1987. 
BARONI URBANI, C.; M. L. DE ANDRADE. The ant genus Proceratium in the extant and fossil record (Hymenoptera: Formicidae). Museo Regionale di Scienze Naturali Monografie (Turin), v.36, p.1-492, 2003.

BOLTON, B. The ant tribe Dacetini. With a revision of the Strumigenys species of the Malagasy Region by Brian L. Fisher, and a revision of the Austral epopostrumiform genera by Steven O. Shattuck. Memoirs of the American Entomological Institute, v.65 (2 vol.), p.1-1028, 2000.

BOLTON, B. An online catalog of the ants of the world. Disponible en http://antcat.org. (visto [07.iii.2015])

BOLTON, B.; B. L. FISHER. Taxonomy of Afrotropical and West Palaearctic ants of the ponerine genus Hypoponera Santschi (Hymenoptera: Formicidae). Zootaxa, v.2843, published 29 Apr. 2011, p.1-118, 2011.

BROWN, W. L., JR. Contributions toward a reclassification of the Formicidae. II. Tribe Ectatommini (Hymenoptera). Bulletin of the Museum of Comparative Zoology, v.118, Published: Jun 1958, p.173-362, 1958.

BROWN, W. L., JR. Contributions toward a reclassification of the Formicidae. III. Tribe Amblyoponini (Hymenoptera). Bulletin of the Museum of Comparative Zoology, v.122, Published: Mar 1960, p.143-230, 1960.

BROWN, W. L., JR. Contributions to a reclassification of the Formicidae. IV. Tribe Typhlomyrmecini (Hymenoptera). Psyche, v.72, Published: 25 Jun 1965, p.65-78, 1965.

BROWN, W. L., JR. Contributions toward a reclassification of the Formicidae. V. Ponerinae, tribes Platythyreini, Cerapachyini, Cylindromyrmecini, Acanthostichini, and Aenictogitini. Search Agriculture (Ithaca, N. Y.), v.5(1), Published: Jun 1975, p.1-115, 1975.

Brown, W. L., Jr. Contributions toward a reclassification of the Formicidae. Part VI. Ponerinae, tribe Ponerini, subtribe Odontomachiti. Section A. Introduction, subtribal characters. Genus Odontomachus. Studia Entomologica, v.19, Published: 30 Dec 1976, p.67-171. 1976.

BROWN, W. L., JR. Contributions toward a reclassification of the Formicidae. Part VI. Ponerinae, tribe Ponerini, subtribe Odontomachiti. Section

B. Genus Anochetus and bibliography. Studia Entomologica, v.20, Published: 30 Aug 1978, p.549$638,1978$.
FEITOSA, R.M.; LACAU, S.; ROCHA, W.D.; OLIVEIRA, A.R.; DELABIE, J.H.C. A giant new arboreal species of the ant genus Anochetus from Brazil (Formicidae: Ponerinae). Annales de la Société Entomologique de France, v. 48, n. 3-4, p. 253-259, 2012.

FERNANDES, I. O.; OLIVEIRA, M. L. DE; DELABIE, J. H. C. Description of two new species in the Neotropical Pachycondyla foetida complex (Hymenoptera: Formicidae: Ponerinae) and taxonomic notes on the genus. Myrmecological News, 19:133$163,2014$.

FERNÁNDEZ, F.; T. M. ARIAS-PENNA. Las hormigas cazadoras en la región Neotropical. In: E. Jiménez, F. Fernández, et al (Ed.). Sistemática, Biogeografía y Conservación de las Hormigas Cazadoras de Colombia. Bogotá D.C., Colombia 622 p.: Instituto de Investigación de Recursos Biológicos Alexander von Humboldt, 2007. Las hormigas cazadoras en la región Neotropical, p.3-39

FERNÁNDEZ, F.; M. OSPINA. Sinopsis de las hormigas de la región Neotropical. In: F. Fernández (Ed.). Introducción a las Hormigas de la Región Neotropical. Bogotá. 424 p.: Instituto Humboldt, Sinopsis de las hormigas de la región Neotropical, p.49-64, 2003.

JAHYNY, B., S. LACAU, et al. Le genre Thaumatomyrmex Mayr 1887, cryptique et prédateur spécialiste de Diplopoda Penicillata. In: E. Jiménez, C. Fernández, et al (Ed.). Sistemática, Biogeografía y Conservación de las Hormigas Cazadoras de Colombia. Bogota, D.C., Colombia. 622 p.: Instituto de Investigación de Recursos Biológicos Alexander von Humboldt, 2007. Le genre Thaumatomyrmex Mayr 1887, cryptique et prédateur spécialiste de Diplopoda Penicillata, p.329-346

KEMPF, W. W. Retoques à classificação das formigas neotropicais do gênero Heteroponera Mayr (Hym., Formicidae). Papéis Avulsos de Zoologia, v.15, Published: 02 Jul 1962, p.29-47, 1962.

KEMPF, W. W. A synopsis of the Neotropical ants of the genus Centromyrmex Mayr (Hymenoptera: Formicidae). Studia Entomologica, v. 9, n. 1-4, p. 401-410, 1967.

KEMPF, W. W. Catálogo abreviado das formigas da região Neotropical. Studia Entomologica, v.15, Published: 25 Aug 1972, p.3-344, 1972.

KUGLER C.; BROWN W. L., JR. Revisionary and other studies on the ant genus Ectatomma, including the description of two new species. Search Agriculture. (Ithaca, N. Y.), v. 24, p. 1-8, 1982. 
Lacau, S.; J. H. C. Delabie. Description de trois nouvelles espèces d'Amblyopone avec quelques notes biogéographiques sur le genre au Brésil (Formicidae, Ponerinae). Bulletin de la Société Entomologique de France, v.107, p.33-41, 2002.

LACAU, S., C. VILLEMANT, et al. Typhlomyrmex Mayr, 1862: un genre meconnu de petites fourmis cryptiques et predatrices (Ectatomminae: Typhlomyrmecini). In: E. Jiménez, F. Fernández, et al (Ed.). Sistemática, Biogeografía y Conservación de las Hormigas Cazadoras de Colombia. Bogotá D.C., Colombia 622 p.: Instituto de Investigación de Recursos Biológicos Alexander von Humboldt, 2008. Typhlomyrmex Mayr, 1862: un genre meconnu de petites fourmis cryptiques et predatrices (Ectatomminae: Typhlomyrmecini), p.239-283.

LACH, L., C. PARR, et al. Ant Ecology. Oxford, UK: Oxford University Press. 2010.

LATTKE, J. E. Studies of neotropical Amblyopone Erichson (Hymenoptera: Formicidae). Contr. Sci. (Los Angel.), v.428, 04Sep 1991, p.1-7. 1991.

LATTKE, J. E. Revision of the ant genus Gnamptogenys in the New World (Hymenoptera: Formicidae). J. Hym. Res., v.4, Published: 25 Sep 1995, p.137-193, 1995.

LATTKE, J. E. Nuevas especies de Gnamptogenys Roger, 1863 de América (Hymenoptera: Formicidae: Ponerinae). Entomotropica, v.17, Agosto 2002, p.135144, 2002.

LATTKE, J. E. Revision of the New World species of the genus Leptogenys Roger (Insecta: Hymenoptera: Formicidae: Ponerinae) Arthropod Syst. Phylog., v.69(3), published online 08.xii.2011, p.127-264, 2011.

LATTKE, J. E., F. FERNÁNDEZ, et al. Identification of the species of Gnamptogenys Roger in the Americas. In: R. R. Snelling, B. L. Fisher, et al (Ed.). Advances in Ant Systematics (Hymenoptera: Formicidae): Homage to E. O. Wilson - $\mathbf{5 0}$ years of Contributions. Gainesville, FL, 690 p.: Memoirs of the American Entomological Institute, vol. 80, 2007. Identification of the species of Gnamptogenys Roger in the Americas, p.254-270
LENHART, P. A.; DASH, S. T.; MACKAY, W. P. A revision of the giant Amazonian ants of the genus Dinoponera (Hymenoptera, Formicidae). Journal of Hymenoptera Research, v. 164, p. 119-164, 2013.

MACKAY, W. P.; E. MACKAY. Revision of the ants of the genus Simopelta Mann. In: E. Jiménez, F. Fernández, et al (Ed.). Sistemática, Biogeografía y Conservación de las Hormigas Cazadoras de Colombia. Bogotá D.C., Colombia 622 p.: Instituto de Investigación de Recursos Biológicos Alexander von Humboldt, 2008. Revision of the ants of the genus Simopelta Mann, p.285-327

MACKAY, W. P.; E. MACKAY. The Systematics and Biology of the New World Ants of the Genus Pachycondyla (Hymenoptera: Formicidae). Lewiston, New York: Edwin Mellen Press. 2010. xii+642 p.

SCHMIDT, C. A.; S. O. SHATTUCK. The higher classification of the ant subfamily Ponerinae (Hymenoptera: Formicidae), with a review of Ponerine ecology and behavior. Zootaxa, v.3817(1), p. 001-242, 2014.

SOSA-CALVO, J.; J. T. LONGINO. Subfamilia Proceratiinae. In: E. Jiménez, F. Fernández, et al (Ed.). Sistemática, biogeografía y conservación de las hormigas cazadoras de Colombia. Bogotá D.C., Colombia 622 p.: Instituto de Investigación de Recursos Biológicos Alexander von Humboldt, 2007. Subfamilia Proceratiinae, p.219-236

WILSON, E. O. Pheidole in the New World: A Dominant, Hyperdiverse Ant Genus. Cambridge, MA: Harvard University Press. 2003. 794 p. 